\title{
Implementación de una Plataforma de Desarrollo CANSAT Multipropósito
}

\author{
Implementation of a Multipurpose CANSAT Development Platform
}

\author{
Leonardo A. Anchino ${ }^{1}$, Andrés F. Torti ${ }^{2}$, Emmanuel M. Dovis ${ }^{3}$, Emanuel Bernardi ${ }^{4}$, Rodolfo Podadera ${ }^{5}$ \\ Grupo de Investigación en Robótica y Control, Universidad Tecnológica Nacional Facultad Regional San Francisco \\ Av. de la Universidad 501, San Francisco. Argentina. \\ ${ }^{1}$ lanchino@sanfrancisco.utn.edu.ar \\ 2 atortiesanfrancisco.utn.edu.ar \\ 3 edovisesanfrancisco.utn.edu.ar \\ 4 ebernardiesanfrancisco.utn.edu.ar \\ ${ }^{5}$ rpodadera@sanfrancisco.utn.edu.ar
}

Recibido: 30/09/19; Aceptado: 14/11/19

\begin{abstract}
This article describes the technical and procedural aspects involved in the implementation of a non-orbital CanSat type nanosatellite. This device integrates mechanical, electronic components and software elements, specifically developed locally to promote scientific-technological dissemination, and the awakening of scientific vocations. Finally, it is important to note that this platform will be used as a development tool in the teaching of space technologies.
\end{abstract}

Keywords: education; nano-satellite; electronics; firmware; space technologies.

Resumen-El presente artículo describe los aspectos técnicos y procedimentales involucrados en la implementación de un nanosatélite no orbital tipo CanSat. Este dispositivo integra componentes mecánicos, electrónicos y elementos de software, desarrollados local y específicamente para fomentar la divulgación científico-tecnológica, y el despertar de vocaciones científicas. Por último, es importante remarcar que la presente plataforma se empleará como instrumento de desarrollo en la enseñanza de tecnologías espaciales.

Palabras clave: educación; nanosatélite; electrónica; firmware; tecnologías espaciales.

\section{INTRODUCCIÓN}

En la actualidad, el ingreso y posterior desgranamiento observado en las carreras de grado, con orientación tecnológica, se ha convertido en un importante obstáculo para el desarrollo de las actividades científicas y técnicas del país. Si bien no es tarea sencilla diagnosticar las razones que ocasionan dicha problemática, es pertinente identificar como causas a la falta de vocación, al desconocimiento y al desinterés en afrontar carreras altamente demandantes [1], [2].

Por tal motivo, desde la presente propuesta, enmarcada en el PID-UTN "Desarrollo de una plataforma educativa basada en proyectos CANSAT" (CCUTNSF0005414) [3], que además constituye parte del proyecto final de grado de dos de los autores [4], se pretende exponer y argumentar las técnicas empleadas en el desarrollo e implementación de un nanosatélite, no orbital, tipo CanSat [5], que se utilizará como instrumento de divulgación de las tecnologías espaciales [6]. El dispositivo desarrollado consiste de un satélite artificial, que se lanza a gran altura para la recolección de datos durante su ascenso y posterior descenso, controlado por un paracaídas.

Es de destacar que si bien la utilización de la plataforma CanSat está ampliamente divulgada [7], [8], e incluso se hallan propuestas con leves variaciones, o nuevos enfoques [9], [10], el objetivo principal de este trabajo consiste en el desarrollo de una plataforma local, del tipo open-source para libre distribución, modificación y reproducción del proyecto (tanto de hardware como de software). La Fig. 1 retrata el satélite CanSat desarrollado.

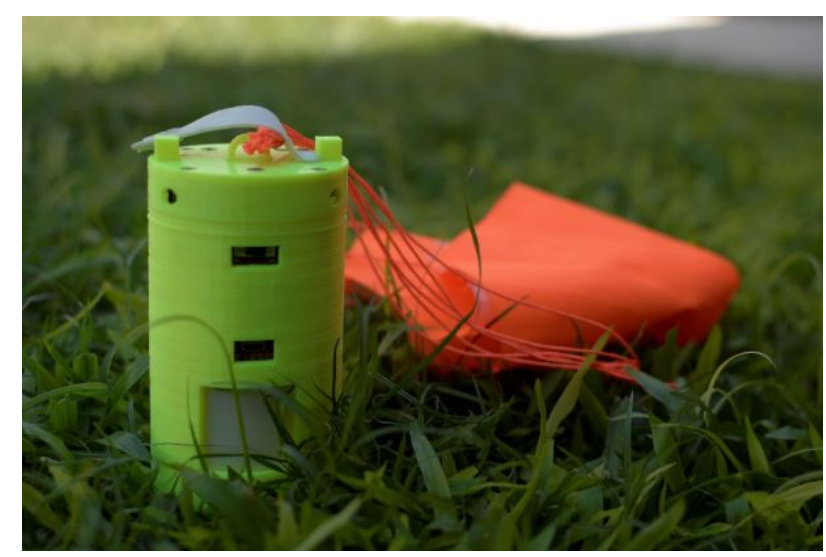

Figura 1: Satélite CanSat.

En la industria satelital, cada satélite puesto en órbita, comercial o no, es proyectado y diseñado en torno a una misión específica. Habitualmente, estas tienen una duración de varios años y dependen de las características del satélite y del objetivo específico de la misión. Además, los satélites cuentan con la capacidad de intercambiar datos de telemetría, e información de la misión, con estaciones terrenas de control. Entre otras capacidades, los satélites científicos a menudo están equipados con diversos sensores para el estudio de fenómenos atmosféricos, la medición de niveles de contaminación, la concentración de gases en la atmósfera, la monitorización de catástrofes naturales, el análisis de cultivos, etc.

Las misiones de la plataforma CanSat son similares a la de un satélite orbital sólo que, en este caso, el mismo 
es no orbital y las misiones duran unos pocos minutos, en lugar de varios años. Para efectuar una misión CanSat, se lo eleva a una altura aproximada de $1000 \mathrm{~m}$, seguido por un descenso controlado donde, en tiempo real, se realiza la recolección de los datos suministrados por los sensores a bordo del satélite. Dicha información se recibe a través de la estación terrena y se visualiza en un programa de computadora, desde el cual se los exporta para un análisis en detalle [11]. Las misiones CanSat, entonces, tienen la misma metodología de planificación que las realizadas en satélites orbitales, permitiendo así que los estudiantes se acerquen a la experiencia de diseñar y desarrollar misiones, para luego proceder con el análisis de los resultados recogidos en vuelo, de manera simple y estructurada. De este modo, es posible involucrar a múltiples asignaturas escolares, tales como matemática, física y ciencias naturales, en el planeamiento de las misiones y en el posterior estudio de los datos obtenidos [7], [8].

Por último, es de destacar que la plataforma desarrollada, forma parte de la propuesta de UNISEC-Argentina [12] para fomentar la enseñanza de tecnologías espaciales, y se utilizó en el dictado del CANSAT Workshop 2019.

\section{INGENIERÍA DE SISTEMA}

Las estrictas especificaciones de los satélites tipo CanSat, según la normativa de la Agencia Espacial Europea, limitan drásticamente su tamaño y peso [13], lo que representa desafíos para utilizar el mismo como plataforma educativa. Un cuidadoso diseño de la electrónica y la mecánica fue requerido para aprovechar al máximo el limitado espacio y permitir, al mismo tiempo, trabajar fácilmente sobre él.

Generalmente, durante la construcción de CanSats se hace uso de la totalidad del espacio disponible, dejando muy pocas opciones para expandir su funcionalidad y haciendo dificultosa la tarea de manipular los componentes internos. Esto trae aparejado que el reemplazar, añadir o quitar componentes resulte una tarea complicada. Habiendo considerado las limitaciones y dificultades de las construcciones tipo CanSat, se planteó que toda la electrónica interna debe poder retirarse fácilmente en una sola pieza para poder trabajar y experimentar cómodamente fuera de la estructura del satélite. La disposición implementada se observa en las Figs. 2 y 3, donde los PCB (del inglés, Printed Circuit Board) fueron apilados con separadores metálicos roscados, mientras que la conexión entre ellos se realizó a través de conectores soldados en cada PCB para evitar la utilización de cables.

Cada uno de los PCBs contiene los diversos módulos y sistemas que componen al CanSat. Comenzando por el superior, la repartición de las placas se da de la siguiente manera:

- PCB_A: módulo de comunicaciones, GPS, HMI y conexionado de servomotores

- PCB_B: administración de batería, reguladores de tensión y protecciones eléctricas

- PCB_C: sistema supervisor, IMU, sensor de presión atmosférica, sensor de humedad, sensor de temperatura

- PCB_D: placa compatible con Arduino ${ }^{\circledR}$ UNO

- PCB_E: placa pre-perforada para experimentación

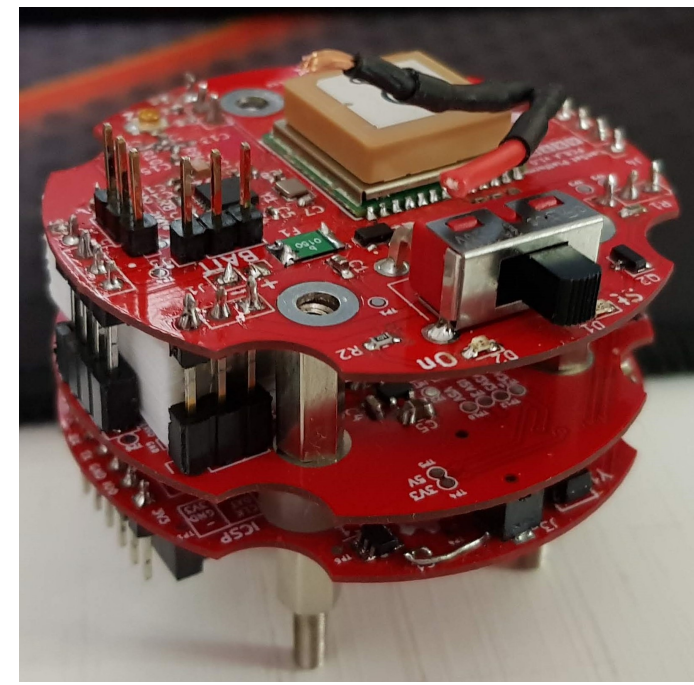

Figura 2: CanSat desarrollado.

El sistema supervisor se encarga de monitorizar no solo las corrientes y tensiones internas, para proteger contra cortocircuitos o sobre-corrientes; sino que también controla en todo momento la altura, velocidad, aceleración y orientación del satélite para actuar en caso de emergencia, por ejemplo, accionando el paracaídas.

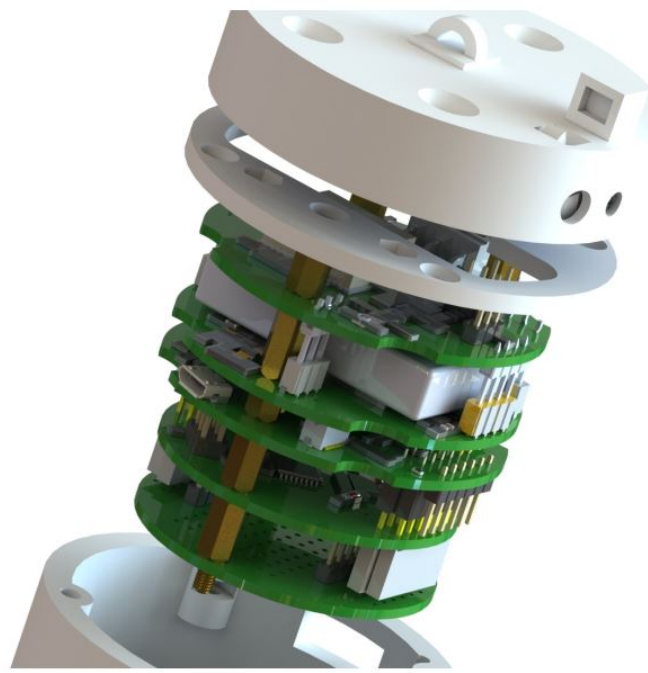

Figura 3: Vista interna del CanSat desarrollado con su respectiva pila de $\mathrm{PCBs}$.

Con respecto a la estructura del CanSat, su diseño fue un hito clave para lograr una buena relación entre la resistencia estructural y el peso que se encuentra limitado a un máximo $350 \mathrm{~g}$. Por razones de simplicidad, se decidió construir la estructura mediante técnicas de impresión 3D, lo que garantiza que pueda ser reproducida fácilmente a un bajo costo. Además, el material plástico otorga un bajo peso ofreciendo, sin embargo, una resistencia mecánica adecuada.

Generalmente, los CanSat son lanzados con un cohete hasta una altitud de aproximadamente $1000 \mathrm{~m}$. Aunque, dado el costo acarreado en su utilización y la dificultad de la oferta local, se previó la posibilidad de elevar el satélite haciendo uso de un globo cautivo o un dron. Puede observarse en la parte superior de la estructura, los mecanismos para la 
liberación y del paracaídas. Por último, en los laterales de la estructura se encuentran cuatro ventanas cubiertas con tapas de Mylar removibles que otorgan la posibilidad de exponer al ambiente cualquier sensor o instrumento que se añada al satélite. Las tapas pueden quitarse, perforarse y/o cortarse a voluntad.

\section{II-A. PCB_A}

Siendo esta la placa soporte para toda la comunicación del CanSat con el mundo exterior, su ubicación en la parte superior fue establecida para disminuir la atenuación de las señales de radiofrecuencia del módulo GPS y del transceptor, encargado del enlace con la estación terrena. La Fig. 4 muestra el PCB en cuestión.

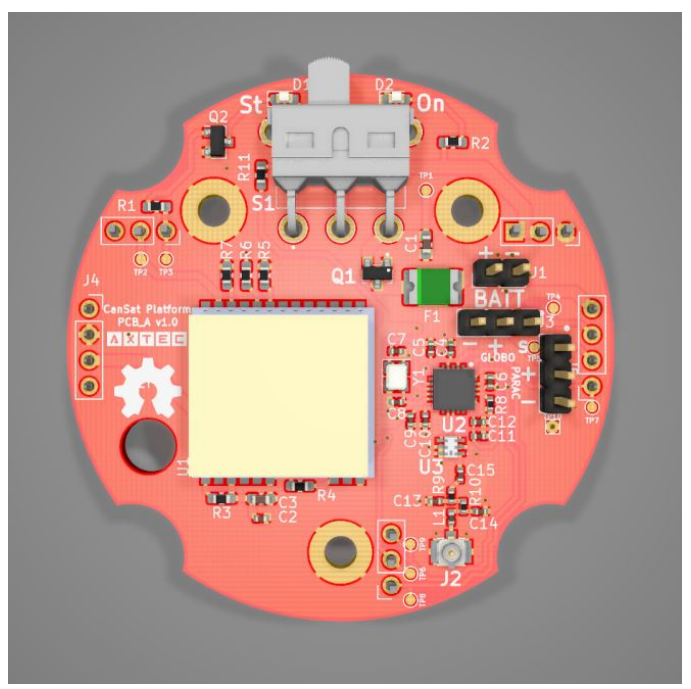

Figura 4: Render del PCB_A.

El transceptor elegido fue el CC1101 [14] de la empresa Texas Instruments ya que integra todos los componentes necesarios para establecer la comunicación con la base de recepción. Los únicos elementos externos necesarios para la comunicación fueron un balún y la antena. En cuanto a su configuración, realizada mediante interfaz SPI, se lo ha programado para trabajar en la banda de libre operación ISM $915 \mathrm{MHz}$.

Con respecto a la antena empleada, se utilizó una antena chip en un PCB aparte que se conecta al transceptor por medio de un cable coaxial RG-178 de $50 \Omega$ de impedancia característica. Éste fue soldado en el extremo del PCB de la antena y conectado por medio del conector U.FL al PCB del CC1101.

El módulo GPS que se eligió fue el A2235-H [15] de la empresa Maestro Wireless que ofrece bajo costo, facilidad de uso, antena pasiva integrada, tamaño reducido y una frecuencia de actualización de $5 \mathrm{~Hz}$. El módulo envía por su interfaz UART comandos NMEA que el sistema supervisor decodifica para obtener la posición y altitud del satélite y determinar además, a partir de estos datos, la velocidad del mismo.

Por su parte, la HMI (del inglés, Human Machine Interface) es muy simple y se compone del interruptor de encendido general y dos LEDs indicadores. Uno de ellos, de color verde, indica cuando hay tensión disponible en el sistema supervisor, mientras que el otro, de color rojo, es controlado por dicho sistema y es usado como un simple indicador de errores o de estado. La descripción detallada de los mismos es enviada a la estación terrena, donde se visualiza a través del software de telemetría.

Dos conectores estándar para servomotores RC están disponibles para la conexión de dos microservos que permiten liberar al satélite desde el globo, dron o cualquier otro medio que efectúe la elevación del mismo, y para liberar el paracaídas durante su descenso. Estos servos son controlados exclusivamente por el sistema supervisor ya que el mismo es el encargado de accionarlos en situaciones de emergencia.

Finalmente, la conexión de la batería se realiza en este PCB mientras que la administración se realiza en la sección siguiente (PCB_B). Aquí, encontramos un fusible del tipo polyfuse que protege a todos los circuitos del satélite en caso de que las demás protecciones fallen y un transistor MOSFET que actúa como protector ante conexiones de polaridad invertida.

\section{II-B. $\quad P C B \quad B$}

En este PCB se encuentran los reguladores para todas las tensiones utilizadas en el satélite así como los circuitos de monitoreo para que el sistema supervisor pueda actuar en consecuencia para proteger al satélite. Tiene un diámetro de $54 \mathrm{~mm}$, se ubica por debajo del PCB_A, regula y controla cinco fuentes de tensión:

- 3,3 V@500 mA: para ser usado por cualquier módulo de expansión agregado al satélite.

- $5 \mathrm{~V} @ 300 \mathrm{~mA}$ : para ser usado por cualquier módulo de expansión agregado al satélite.

- 3,3 V@150 mA (internos): esta tensión es usada por el sistema supervisor y los sensores indispensables para el funcionamiento del satélite. Tener una tensión separada permite que por más que exista algún problema en la línea de $3,3 \mathrm{~V}$, utilizada para los módulos de expansión, el sistema supervisor seguirá funcionando.

- $5 \mathrm{~V} @ 200 \mathrm{~mA}$ (internos): esta tensión es usada para accionar a los servos en el PCB_A. Al ser una tensión separada permite accionar los mismos por más que existan problemas en la línea de $5 \mathrm{~V}$ que se utiliza para los módulos de expansión.

- Batería: tensión sin regular proveniente de la batería del satélite, sólo se encuentra limitada en corriente y su tensión puede variar entre $3,5 \mathrm{~V}$ y $4,2 \mathrm{~V}$.

La fuente de $3,3 \mathrm{~V}$ está compuesta por un convertidor buck ADP2108AUJ-3.3 [16] de la empresa Analog Devices que se encarga de la regulación de la tensión. Si bien la corriente máxima que es capaz de entregar es de $600 \mathrm{~mA}$, esta es limitada por el sistema supervisor a $500 \mathrm{~mA}$. Para medir la corriente que la fuente entrega se utiliza una resistencia shunt de $120 \mathrm{~m} \Omega$ cuya caída de tensión es amplificada por el circuito integrado INA194 [17] de la empresa Texas Instruments, utilizando una ganancia de cincuenta veces la tensión de entrada. El fabricante recomienda que la resistencia genere una caída de tensión entre $50 \mathrm{mV}$ y $100 \mathrm{mV}$ para obtener los mejores resultados. En este caso la caída de tensión máxima es de $60 \mathrm{mV}$, generando una tensión de salida máxima de $3 \mathrm{~V}$, con una tensión de referencia en el ADC de $3,3 \mathrm{~V}$. 


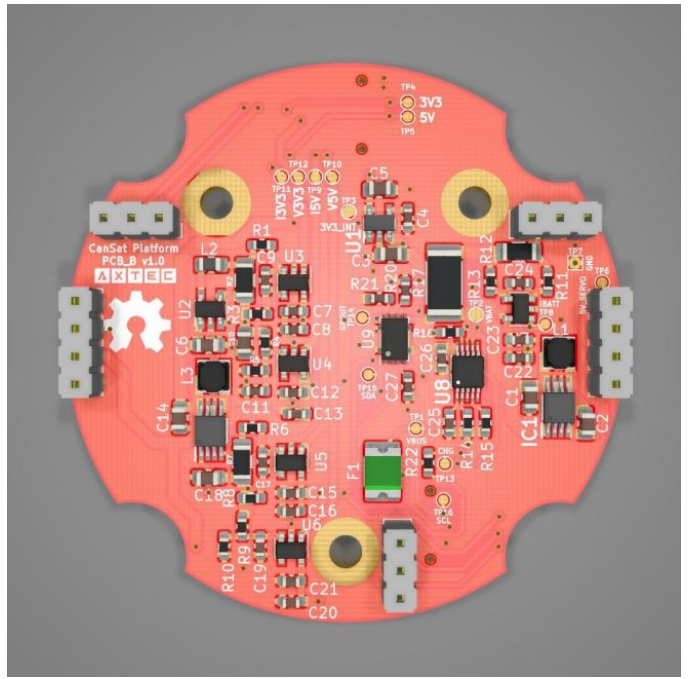

Figura 5: Render del PCB_B.

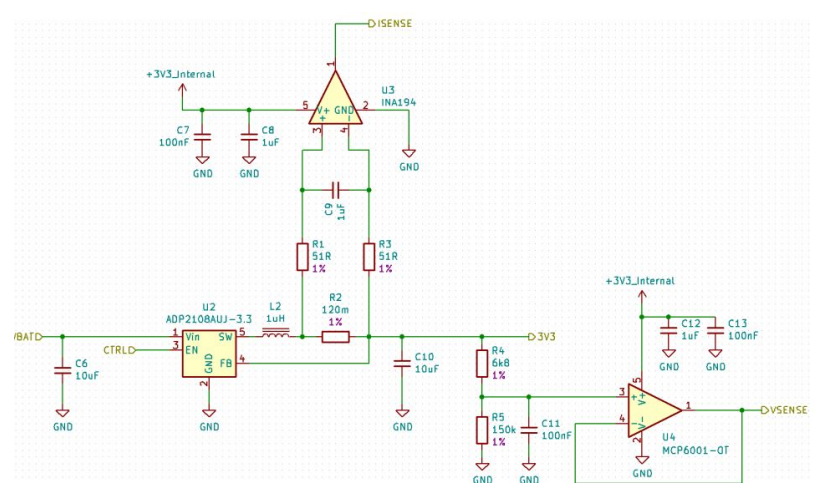

Figura 6: Esquemático de la fuente de $3,3 \mathrm{~V}$ implementada.

Despreciando los efectos de la temperatura sobre la resistencia, la misma tiene una tolerancia del $1 \%$, por lo que valor puede encontrarse entre $118,8 \mathrm{~m} \Omega$ y $121,2 \mathrm{~m} \Omega$. Por otro lado, la ganancia del INA194 tiene también una tolerancia del $1 \%$, haciendo que la misma varíe entre 49,5 y 50,5 . Esto produce una banda de tolerancia en la tensión de salida del INA194 del $2 \%$ o $10 \mathrm{~mA}$. A diferencia de las variaciones por temperatura, estas tolerancias se compensan fácilmente midiendo los valores de la resistencia y la ganancia del amplificador a temperatura ambiente. Por otro lado, se calcula la resolución teórica de la corriente medida sabiendo que la resolución del ADC es de 10 bits. Debido a que la tensión máxima de salida no concuerda con el fondo de escala, el número de cuentas $n$ disponibles se reduce a 931, por lo que la resolución teórica de corriente, entonces, es de $537 \mu \mathrm{A}$.

Ya que la tensión de referencia del $\mathrm{ADC}$ es de $3,3 \mathrm{~V}$ se conecta la fuente directamente su entrada. Sin embargo, bajo este supuesto el dispositivo sería incapaz de detectar situaciones de sobre-tensión donde la misma supere los $3,3 \mathrm{~V}$. Por eso, se utiliza un divisor resistivo compuesto por R4 y R5, ambas con una tolerancia del $1 \%$. La tensión de salida obtenida es de $3,16 \mathrm{~V}$. Esta relación permite medir tensiones de hasta $3,45 \mathrm{~V}$.

Si la tensión de salida de la fuente supera dicho voltaje, no se podría medir pero sí se habría detectado que existe una sobre-tensión y la misma será limitada por la tensión de salida máxima del amplificador operacional rail-to-rail $\mathrm{U} 4$, protegiendo, de este modo, la entrada del ADC.

La fuente de $5 \mathrm{~V}$ está compuesta por un convertidor boost MCP1642 de la empresa Microchip que eleva la tensión de batería y, a pesar de que el sistema supervisor limita la corriente a $300 \mathrm{~mA}$, es capaz de suministrar aproximadamente $10 \mathrm{~A}$ de corriente con $3,6 \mathrm{~V}$ de entrada.

El circuito utilizado es muy similar a la fuente de $3,3 \mathrm{~V}$, variando únicamente los valores de la resistencia shunt y el divisor resistivo para la medición de tensión. La primera se fijó en $200 \mathrm{~m} \Omega$ para que produzca una tensión de salida máxima de $3 \mathrm{~V}$, bajo una corriente máxima de $6 \mathrm{~mA}$, y con una resolución de $322 \mu \mathrm{A}$. El segundo se fijó para que la tensión entregada fuese de $3,2 \mathrm{~V}$, otorgando al ADC una tensión máxima de entrada fuera de la saturación de $5,16 \mathrm{~V}$. La tolerancia quedó fijada, nuevamente, en $2 \%$, mientras que la resolución del ADC permite diferenciar pasos de $5,04 \mathrm{mV}$.

Las denominadas fuentes internas son aquellas de $5 \mathrm{~V}$ y $3,3 \mathrm{~V}$ utilizadas exclusivamente por los sistemas base del satélite y que no pueden ser accedidas por los módulos de expansión. Esta situación garantiza que el sistema supervisor, los sensores y los mecanismos de liberación sigan funcionando por más de que existan cortocircuitos o sobrecorrientes en las fuentes que alimentan los módulos de expansión, asegurando la integridad del satélite.

En este PCB se encuentra también todo el sistema de administración de batería que se encarga de medir el estado de carga, la corriente que circula y realiza la carga de la misma. La batería utilizada en el satélite es de tipo Li-Po con una capacidad de $600 \mathrm{mAh}$. Para su carga se eligió el circuito integrado BQ24091 [18] de la empresa Texas Instruments, el cual lleva a cabo su tarea de manera totalmente autónoma.

Asimismo, se implementó el circuito integrado BQ27441 [19] de la empresa Texas Instruments como fuel gauge. Éste incorpora todo lo necesario para medir el estado de carga de la batería, requiriendo únicamente una resistencia shunt externa para la medición de la corriente que circula desde y hacia la batería. Todos los datos reportados por el integrado son adquiridos a través de la interfaz I2C desde la cual se leen parámetros como capacidad nominal de la batería, capacidad restante, corriente promedio, tensión de la batería, potencia promedio, etcétera.

\section{II-C. $\quad P C B \_C$}

En este PCB se encuentra el microcontrolador del sistema supervisor, los sensores asociados, que junto con el módulo GPS del PCB_A permiten al sistema determinar los diversos parámetros del satélite en todo momento, y el conector de expansión con sus protecciones, que será utilizado por todos los módulos de expansión que se conecten al satélite.

II-Cl. Microcontrolador: El microcontrolador principal elegido fue el ESP32 [20] de la empresa Espressif soldado en un PCB propio, listo para ser utilizado. La razón por la que se decantó por el módulo y no por el microcontrolador en su encapsulado original es que el primero incorpora la memoria flash externa necesaria por el microcontrolador, el cristal, la antena para Wi-Fi y Bluetooth y todos los componentes pasivos necesarios (además de contar con 


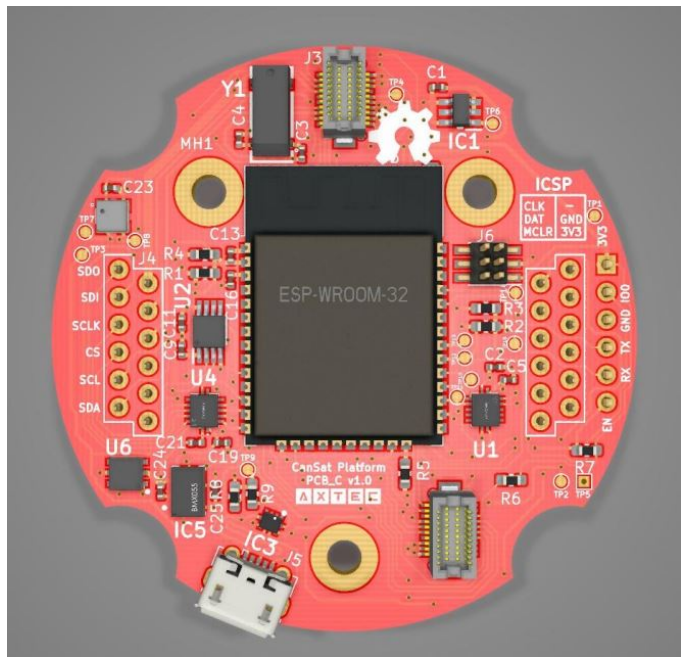

Figura 7: Render del PCB_C.

certificación de la FCC), todo esto a un precio menor que si se adquirieran los componentes por separado.

Con respecto al ADC con el que cuenta el ESP32, se ha probado que su comportamiento es muy poco lineal y, también, existe escasa documentación sobre sus características aparte de su resolución. Es por ello que se recurrió a un microcontrolador PIC16LF18325 [21] de la empresa Microchip para realizar las lecturas de las tensiones. Un ADC externo con bus I2C podría haber sido utilizado, pero debido a la disponibilidad se optó por este microcontrolador.

Este microcontrolador se alimenta desde una referencia de voltaje MCP1501T [22], también de la empresa Microchip, que le provee una tensión de $3,3 \mathrm{~V}$ estable para ser usada por el módulo ADC. La razón para usar una tensión separada es que el regulador interno de $3,3 \mathrm{~V}$, compuesto por el integrado MIC5323, tiene una tolerancia de $2 \%$ en su tensión de salida, sumado a todo el ruido eléctrico producido por los sistemas digitales que utilizan dicho bus de alimentación. El MCP1501T, por otro lado, tiene una tolerancia de $0,1 \%$, bajo nivel de ruido, se encuentra conectada únicamente al microcontrolador que realiza las mediciones y tiene un coeficiente de temperatura máximo de $50 \mathrm{ppm} /{ }^{\circ} \mathrm{C}$, lo cual es importante debido a que el satélite puede experimentar grandes cambios de temperatura durante su ascenso y descenso.

Retornando al ESP32, el desarrollo de su firmware fue realizado mediante el framework otorgado por el fabricante ESP-IDF: Espressif IoT Development Framework, haciendo uso del RTOS9 FreeRTOS y el lenguaje C. Este microcontrolador tiene varias tareas críticas y el RTOS da la posibilidad de asignarles prioridades, asegurando que las de mayor prioridad nunca sean interrumpidas por las de menor prioridad. Las funciones que debe realizar el mismo son:

- Detectar cortocircuitos, sobre-corrientes y sobretensiones en las fuentes de alimentación utilizadas por lo módulos de expansión y actuar en consecuencia.

- Comprobar el correcto funcionamiento de los sensores del satélite.

- Comunicarse con la estación terrena y realizar el envío de los parámetros del satélite.

- Abrir el paracaídas cuando la altura es peligrosamente baja y no se ha accionado.

- Liberar el satélite del globo, dron o cualquier medio que realice la elevación del mismo al llegar a la altura designada.

II-C2. Sensores: El sensor elegido para monitorear la temperatura y humedad externa es el Si7006 [23] de la empresa Silicon Labs. Este integrado, de bajo consumo y reducido tamaño, utiliza el bus I2C para reportar los datos medidos al microcontrolador principal. Cabe destacar que se escogió la versión del sensor que viene con una lámina PTFE protectora, en la parte superior, que impide el ingreso de agua o partículas que rápidamente ocasionarían que los reportes de humedad fueran viciados.

El sensor de presión atmosférica implementado fue el MS563702BA03-50 [24] de la empresa TE Connectivity. Cuenta con un sensor de presión barométrica ultra compacto con dimensiones de $3 \mathrm{~mm} \times 3 \mathrm{~mm} \times 1 \mathrm{~mm}$ y muy bajo consumo. Su alimentación es en base a una tensión de $3,3 \mathrm{~V}$ y su comunicación, mediante interfaz I2C. Contiene además un sensor de temperatura que permite compensar las mediciones de presión.

Por último, el IMU, que permite conocer no solo la aceleración que experimenta el satélite sino también su orientación en el espacio, es el BMX055 [25] de la empresa Bosch. Dentro de sus características principales se destacan la comunicación I2C, su giroscopio de 16 bits, acelerómetro de 12 bits y $\pm 2 \mathrm{~g}$ o $\pm 16 \mathrm{~g}$ (el parámetro g se corresponde con $9,8 \mathrm{~m} \mathrm{~s}^{-2}$ ), sensor geomagnético de 3 ejes, bajo consumo y tamaño compacto.

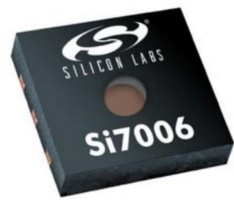

(a) Si7006.

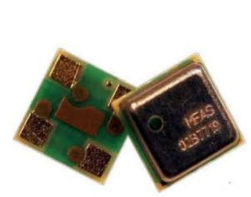

(b) MS563702BA03.

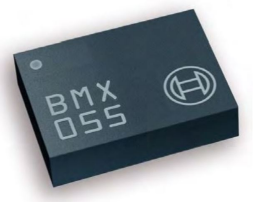

(c) BMX055
Figura 8: Sensores del PCB_C.

\section{II-D. PCB_D}

Este PCB es equivalente y compatible con la plataforma Arduino ${ }^{\circledR}$ UNO. El conector micro-USB incluido permite comunicarse con el bootloader a través del único puerto UART disponible, el cual es, a su vez, utilizado para la comunicación con el sistema supervisor. Así, como todos los módulos de expansión que se conecten, el mismo contiene un conector de expansión y un diámetro de $47 \mathrm{~mm}$.

Su utilización en el CanSat fue orientado para que los estudiantes puedan realizar sus propios programas para la misión utilizando una plataforma de desarrollo sencilla y conocida, tal como lo es Arduino ${ }^{\circledR}$, dejando de lado la operación sobre el ESP32 y el riego que esto conlleva para la seguridad del CanSat.

El Arduino ${ }^{\circledR}$ UNO implementado en la plataforma es ligeramente distinto en las conexiones de sus pines. Sólo cuatro entradas analógicas están disponibles ya que las dos restantes se utilizan en el bus I2C; el pin D7 es interno únicamente y se encuentra conectado al LED; y los pines D8 y D9 no se encuentran implementados. 


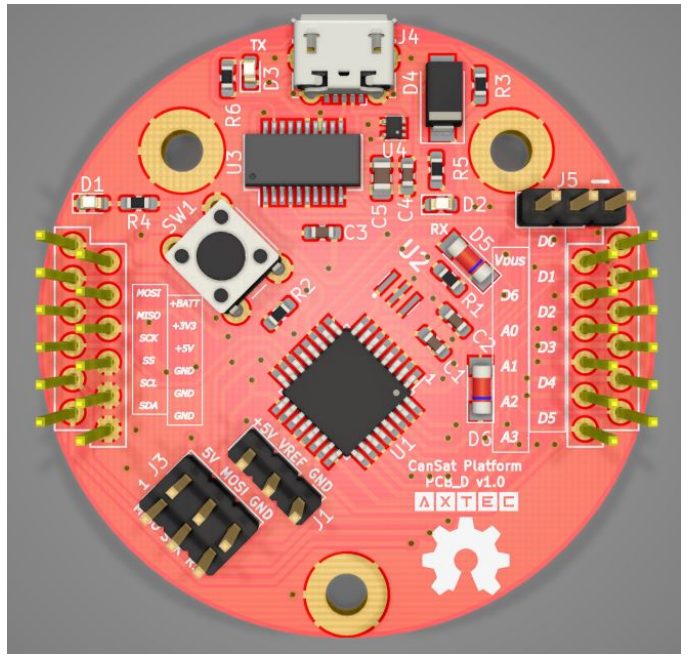

Figura 9: Render del PCB_D.

Tanto en este PCB como en el PCB_C visto en la sección anterior, se encuentra el conector de expansión que es utilizado para añadir módulos de expansión a la plataforma. La Fig. 10 muestra las conexiones del mismo.
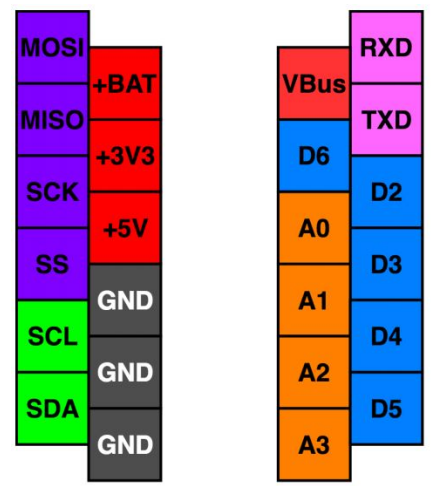

Figura 10: Conectores de expansión.

\section{II-E. PCB_E}

Este PCB es otro módulo de expansión por lo que diámetro es de $47 \mathrm{~mm}$ y contiene el conector de expansión descrito anteriormente. El módulo es una placa pre-perforada que permite armar circuitos simples para poder experimentar. Se da acceso a todos los pines del conector de expansión para poder utilizar cables y realizar conexiones simples y rápidas.

\section{II-F. PCB Estación Terrena}

La estación terrena es el medio por el cual el software de telemetría en el PC se comunica con el satélite en todo momento. Cuenta con una conexión USB y conector SMA para conectar la antena que se considere adecuada para la misión a realizar.

El transceptor utilizado es el mismo que el que se usó en el PCB_A, siendo el circuito utilizado idéntico con la excepción de que en este caso se utiliza un conector tipo SMA en lugar de U.FL. La interfaz entre el bus SPI del transceptor y la conexión USB de la PC se realizó por medio del circuito integrado MCP2210 [26] de la empresa

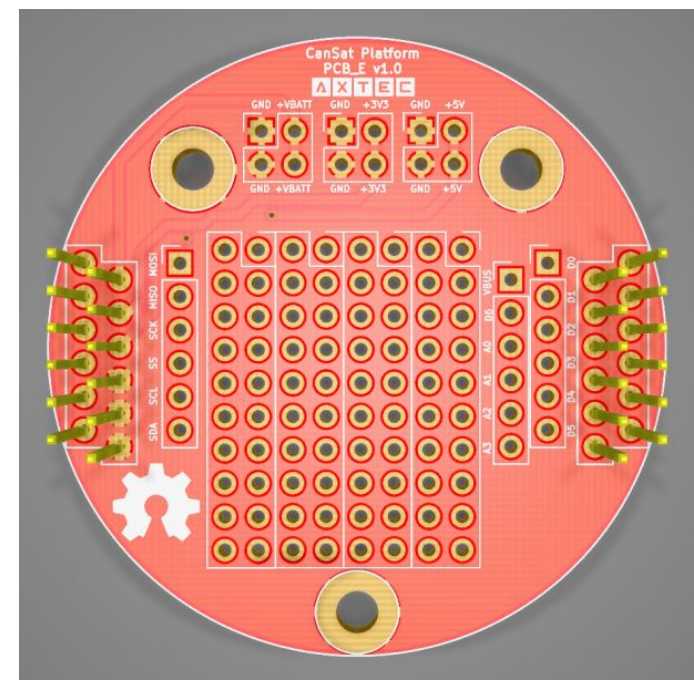

Figura 11: Render del PCB_E.

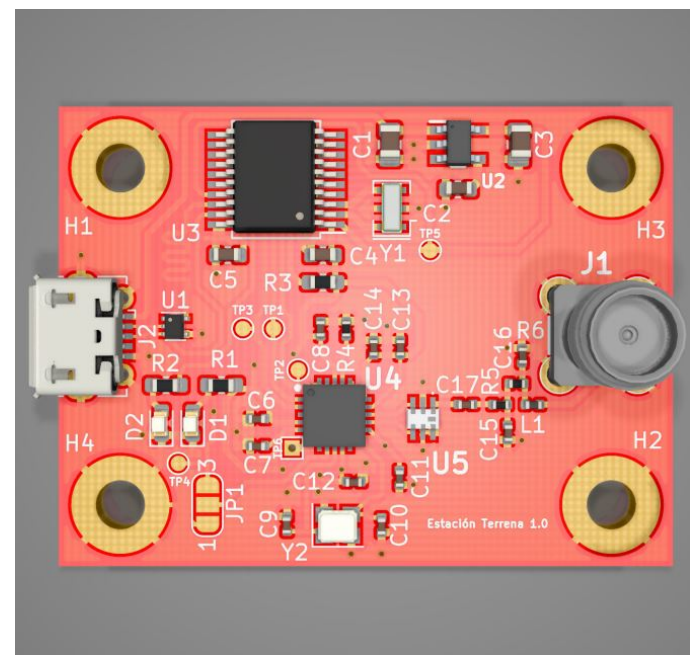

Figura 12: Render del PCB de la estación terrena.

Microchip, que permite enviar y recibir datos por el bus SPI a partir de una conexión USB controlada desde la PC.

\section{II-G. Software de telemetría}

La plataforma cuenta con un software de telemetría que se encarga de administrar todos los recursos relacionados al CanSat. Para ello es necesario conectar la estación terrena a una computadora por medio de un puerto USB para permitir a la aplicación establecer la comunicación con el satélite. La estación terrena queda entonces compuesta por:

1. Hardware con enlace de radio en la banda ISM $915 \mathrm{MHz}$

2. Aplicación para computadora (Software de telemetría)

La aplicación creada cuenta con una interfaz simplificada, donde el espacio de trabajo se divide en tres grandes sectores:

- Barra superior de funciones generales: incluye funciones como mostrar/ocultar menú desplegable para agrandar el panel de acciones, ver notificaciones recibidas y cambiar idioma utilizado en la interfaz

- Menú desplegable de funciones específicas: en él se pueden acceder a las características relacionadas a la 


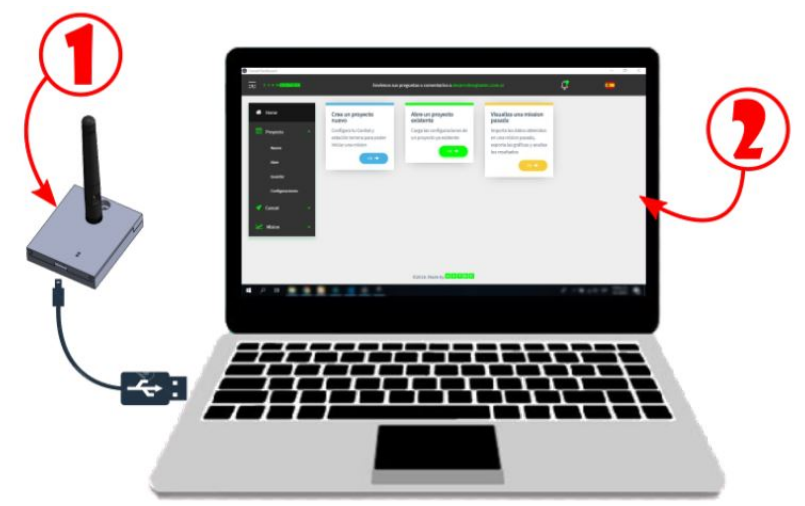

Figura 13: Estación terrena.

gestión del proyecto, administración, monitoreo del satélite y el módulo de misiones

- Panel de acciones: espacio reservado para mostrar el contenido relacionado a la función actual seleccionada

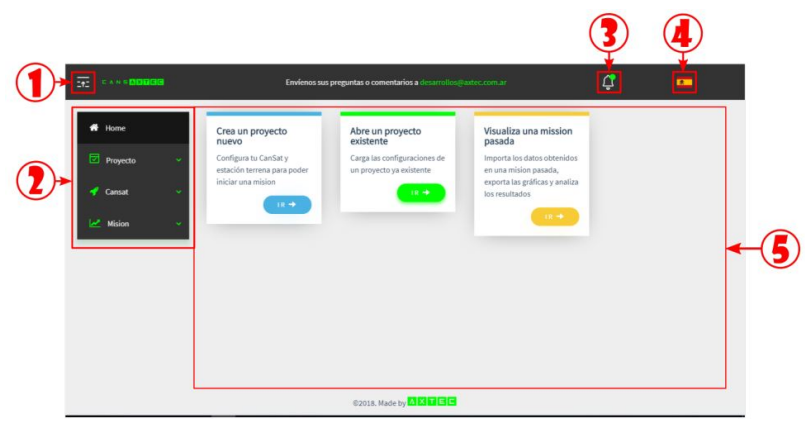

Figura 14: Página inicial de la aplicación. Botón para mostrar/ocultar el menú (1), menú de funciones (2), botón de notificaciones (3), botón de idiomas (4) y panel de acciones (5).

El software se dividió en múltiples módulos para facilitar el desarrollo. Se cuenta con cinco módulos interconectados:

1. Módulo de proyecto

2. Módulo de CanSat

3. Módulo de misiones

4. Módulo de comunicaciones

5. Módulo de alertas

El primer módulo es el encargado de la lógica de creación, validación, guardado y cargado de proyectos. Para esto, se almacenan en un archivo todas las configuraciones imprescindibles para realizar una correcta comunicación, tales como el tipo de protocolo y las protecciones más importantes. Cada archivo de proyecto generado posee la extensión .cansat_pro y solo podrá ser reconocido e interpretado por el software de PC. Para facilitar la creación y configuración, el usuario será guiado, a través de la interfaz, por un asistente.

El módulo de CanSat es el responsable de mostrar los estados del CanSat asociado a una estación terrena, valores de sensores, configuración de protecciones y pruebas de integridad. Las funciones asociadas a este módulo son las siguientes:

- Función enlace: muestra los estados relacionados a la comunicación de radio entre la estación terrena y el satélite
- Prueba de integridad: comprueba todos los componentes internos del CanSat para asegurar un buen funcionamiento

- Protecciones: configura valores seguros de trabajo en las distintas variables a medir y genera alertas en caso de activación de una protección eléctrica

- Función de sensores: realiza peticiones sobre los estado de todos los sensores montados en el CanSat

El módulo de misiones posibilita la creación y visualización de misiones. En la creación de una misión, se seleccionan qué variables se desean graficar, si las que trae por defecto el satélite o nuevas añadidas en las placas de expansión.

Los datos recolectados en tiempo real son guardados en un archivo con extensión .cansat_data. Esto permite su posterior análisis mediante la importación de una misión pasada o exportado en otros formatos.

Por su parte, el módulo de comunicaciones es el intermediario en la comunicación con el satélite, decodifica los paquetes y toma acciones acorde a ellos. El usuario sólo interacciona con este módulo a través de las notificaciones y/o alertas en caso de existir algún aviso de protección eléctrica. Para la correcta interpretación de la comunicación y validación de los datos recibidos entre el satélite y la estación terrena, fue necesario la implementación de un protocolo de comunicación dedicado, similar a los utilizados en los módulos XBee de la empresa Digi ${ }^{\circledR}$ en su modo API [27].

Por último, el módulo de alertas es el encargado de mostrar notificaciones al usuario, dependiendo de la situación que se presente. Cuando una alerta es desplegada en la interfaz, se bloquean todas las demás funciones ya que se requiere que el usuario responda a la misma. El usuario tendrá una pantalla especial donde se mostraran las distintas notificaciones que fue recibiendo desde que está utilizando el programa en orden cronológico permitiendo tener un reporte más sobre su actividad en la plataforma.

\section{RESUlTADOS}

Se ha ensayado la plataforma a través de múltiples lanzamientos, de hasta $150 \mathrm{~m}$ de altura, con resultados como los que se bosquejan en la Fig. 15. Estos ensayos permitieron evaluar, satisfactoriamente, la robustez estructural, el funcionamiento del sistema y el cumplimiento de las normas impuestas por la Agencia Espacial Europea [13].

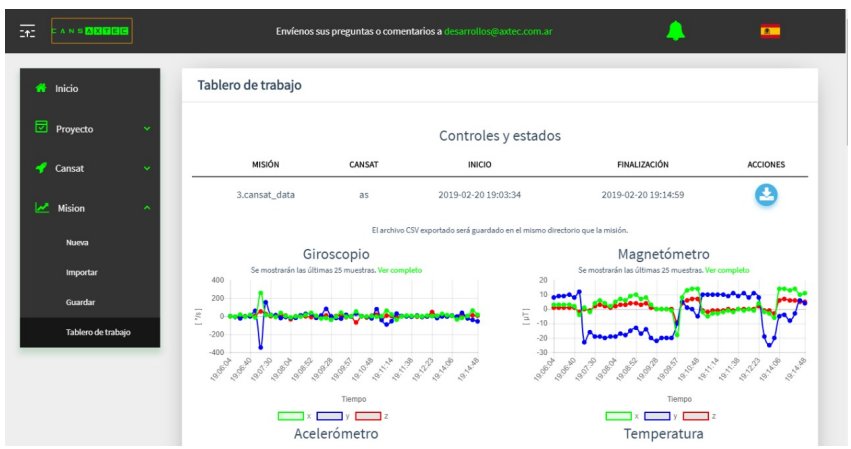

Figura 15: Resultados de una misión. 


\section{CONCLUSión}

En base a los resultados expuestos en la sección previa, es posible afirmar que se han cumplido los objetivos inicialmente propuestos. En resumen, se ha construido una plataforma de desarrollo, lista para usar, basada en un satélite CanSat, cuya operación es sencilla, y de diseño libre y abierto.

Las pruebas realizadas expusieron unos pocos aspectos de hardware a mejorar en la siguiente versión, que se encuentra en desarrollo. Además, se planea ensayar la plataforma a su altura máxima de diseño $(1000 \mathrm{~m})$ que no fue alcanzada por falta de medios de elevación.

Por último, ya que el código y los diseños se han liberado bajo licencia GNU GPL Versión 3 [28], se espera que este trabajo sirva como puntapié inicial para aquellas personas que deseen contribuir al proyecto.

\section{AgRAdecimientos}

Los autores desean agradecer a los docentes y directivos de la institución de nivel medio IPET $\mathrm{N}^{\mathrm{0}} 50$, de la ciudad de San Francisco (Córdoba), por su apoyo, colaboración y predisposición en las actividades de divulgación.

\section{REFERENCIAS}

[1] UTN-BA. Esfuerzos en todo el país para promover el ingreso a la UTN. [Online]. Available: https://www.frba.utn.edu.ar/esfuerzos-entodo-el-pais-para-promover-el-ingreso-a-la-utn/

[2] ITBA. Apostar a las ingenierías, las carreras del futuro. [Online]. Available: https://www.itba.edu.ar/la-universidad/prensa/apostara-las-ingenierias-las-carreras-del-futuro/

[3] L. Anchino, A. Torti, M. Miretti, E. Bernardi, G. Peretti, and R. Podadera, "Desarrollo de un dispositivo CANSAT para fomentar el acceso a las tecnologías espaciales," in WICC2019. San Juan, Argentina: Red de Universidades Nacionales con Carreras de Informática (RedUNCI), 04 2019, pp. 971-974.

[4] L. Anchino and A. Torti, "Desarrollo de satélite cansat para utilizar como plataforma educativa y fomentar el interés en ingenierías," Proyecto final de carrera, Universidad Tecnológica Nacional, 032019.

[5] E. S. Agency. (2019, 02) What is a CanSat? [Online]. Available: https://www.esa.int/Education/CanSat/What is a CanSat

[6] A. Torti, L. Anchino, L. Chiappero, M. Miretti, E. Dovis, E. Bernardi, J. Calloni, J. Tomé, M. A. Berón, and R. Podadera, "Estrategia de Divulgación y Enseñanza para Fomentar el Interés en las Tecnologías Espaciales," in WICC2019. San Juan, Argentina: Red de Universidades Nacionales con Carreras de Informática (RedUNCI), 042019 , pp. 601-604.

[7] Y. Miyazaki and M. Yamazaki, "A practical education of space engineering by using Cansat and pico-satellite-Fruitful collaboration with UNISEC for success of student satellite program," in 2013 6th International Conference on Recent Advances in Space Technologies (RAST). IEEE, 2013, pp. 1081-1086.
[8] H. Aly, O. Sharkawy, A. Nabil, A. Yassin, M. Tarek, S. M. Amin, and M. K. Ibrahim, "Project-based space engineering education: Application to autonomous rover-back CanSat," in 2013 6th International Conference on Recent Advances in Space Technologies (RAST). IEEE, 2013, pp. 1087-1092.

[9] M. O. Kizilkaya, A. E. Oguz, and S. Soyer, "CanSat descent control system design and implementation," in 2017 8th International Conference on Recent Advances in Space Technologies (RAST), June 2017, pp. 241-245.

[10] I. Aliyev, C. A. Misirli, S. Ozturk, E. Mahmurat, A. Erkek, S. Kok, D. Kocyigit, S. Uzun, and R. A. Vural, "Design of solar powered subscale glider for CanSat competition," in 2017 8th International Conference on Recent Advances in Space Technologies (RAST), June 2017, pp. 453-457.

[11] M. E. Aydemir, R. C. Dursun, and M. Pehlevan, "Ground station design procedures for CANSAT," in 2013 6th International Conference on Recent Advances in Space Technologies (RAST), June 2013, pp. 909-912.

[12] U. Global. UNISEC Argentina Point of Contact. [Online]. Available: http://www.unisec-global.org/reyna.html

[13] E. S. Agency, "2019 CanSat Guidelines," 2019

14] T. Instruments. Low-Power Sub-1 GHz RF Transceiver. [Online]. Available: http://www.ti.com/lit/ds/symlink/cc1101.pdf

[15] M. Wireless. Maestro GPS receiver datasheet. [Online]. Available: http://update.maestro-wireless.com/GNSS/A2235H/Maestro_A2135_HA2235_H_v11.pdf

[16] A. Devices. Compact, $600 \mathrm{~mA}, \quad 3 \mathrm{MHz}$ Step-Down DC-to-DC Converter. [Online]. Available: https://www.analog.com/media/en/technical-documentation/datasheets/ADP2108.pdf

[17] T. Instruments. INA19x Current Shunt Monitor -16 V to $+80 \mathrm{~V}$ Common-Mode Range. [Online]. Available: http://www.ti.com/lit/ds/symlink/ina193.pdf

[18] T. Instruments. bq2409x 1A, Single-Input, Single Cell Li-Ion and Li-Pol Battery Charger. [Online]. Available: http://www.ti.com/lit/ds/symlink/bq24095.pdf

[19] T. Instruments. bq27441-G1 System-Side Impedance Track $^{\mathrm{TM}}$ Fuel Gauge. [Online]. Available: http://www.ti.com/lit/ds/symlink/bq27441-g1.pdf

[20] Espressif. ESP32 Overview. [Online]. Available: https://www.espressif.com/en/products/hardware/esp32/overview

[21] Microchip. PIC16(L)F18325/18345. [Online]. Available: http://ww1.microchip.com/downloads/en/devicedoc/40001795e.pdf

[22] - MCP1501T. [Online]. Available: http://ww1.microchip.com/downloads/en/DeviceDoc/20005474E.pdf

[23] S. Labs. Si7006-A20. [Online]. Available: https://www.silabs.com/documents/public/data-sheets/Si7006A20.pdf

[24] T. Connectivity. 2 BAR INDUSTRIAL ALTIMETER SENSOR. [Online]. Available: https://www.te.com/usa-en/product-MS563702BA0350.html

[25] Bosch. BMX055, Absolute Orientation Sensor. [Online]. Available: https://www.bosch-sensortec.com/bst/products/all_products/bmx055

[26] Microchip. USB-to-SPI Protocol Converter with GPIO (Master Mode). [Online]. Available: http://ww1.microchip.com/downloads/en/devicedoc/22288a.pdf

[27] Digi. Api frame specifications. [Online]. Available: https://www.digi.com/

[28] L. Anchino and A. Torti. Plataforma de divulgación CANSAT (AT-SAT). [Online]. Available: https://github.com/dragondgold/AtSat 